Dept. of Disease Reproductive, Brucella Lab.,

Animal Reproduction Research Institute, Pyramids, Giza, Egypt.

\title{
INDIRECT AND COMPETITIVE ELISA AS A TOOL FOR DIAGNOSIS OF BRUCELLOSIS IN VACCINATED AND INFECTED CATTLE
}

(With 3 Tables)

\author{
By \\ M.M. BASSIONY; I.G.A. IBRAHIM \\ and EMAN S. RAMADAN
}

(Received at

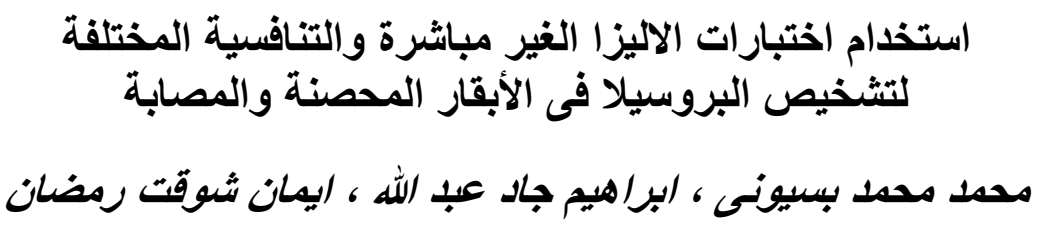

فى هذه الدر اسة تمت مقارنة نتائج اختبار الاليزا الغير مباشر و الاليزا التنافسى بنتائج الاختبار ات

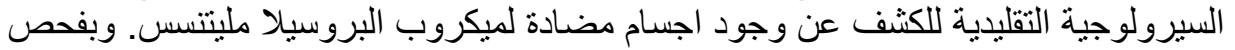

360 عينة سيرم لحيو انات مصابة وغير محصنة أشارت النتائج الي أن حساسية اختبار الاليزا

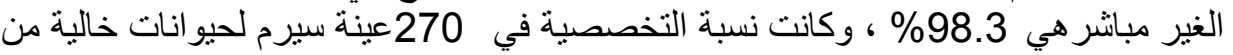

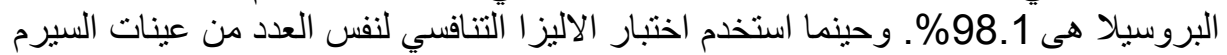

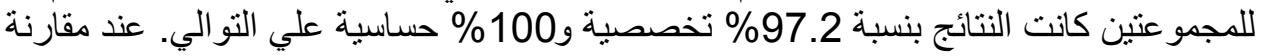

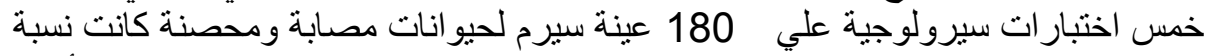

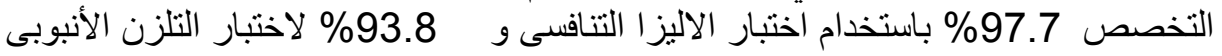

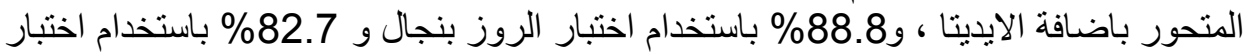

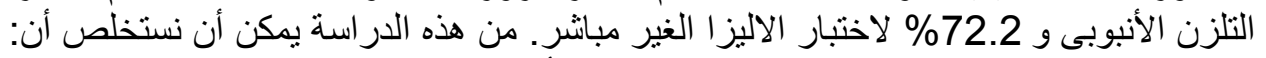

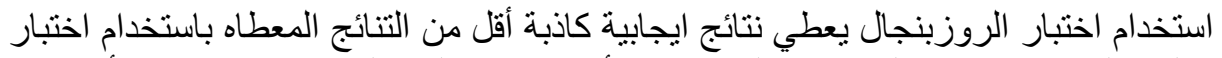

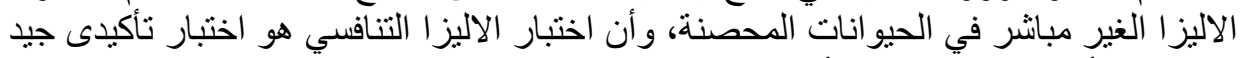

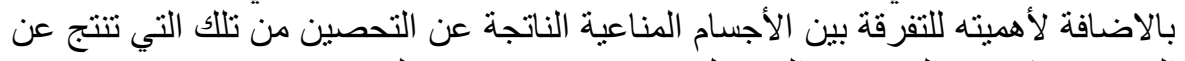

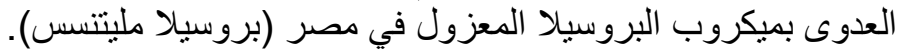

\section{SUMMARY}

The results of an indirect ELISA (iELISA) and a competitive ELISA (cELISA) for detection of antibody to Brucella melitensis in cows were compared with those of conventional serological tests. The sensitivity of iELISA using 360 sera from infected not vaccinated animals was $98.3 \%$ and the specificity in 270 sera from brucellosis-free animals was $98.1 \%$. The cELISA when tested in the same numbers of sera of the two groups 
were $97.2 \%$ Specific and $100 \%$ sensitive respectively. When comparing five serological tests on 180 sera from vaccinated-infected animals, the specificity was $97.7 \%$ for cELISA, $93.8 \%$ for EDTA-modified SAT, 88.8 $\%$ for RBPT, $82.7 \%$ for SAT and $72.2 \%$ for iELISA. It can be concluded from this study that the RBPT gave less false positive results than the iELISA in vaccinated animals and the cELISA is a good confirmatory test with the advantage of distinguishing the antibody response due to vaccination from that resulting from infection with the local Brucella isolates(Brucella melitensis) in Egypt.

Key words:

\section{INTRODUCTION}

Serological detection of antibodies is usually the method of choice for control and eradication of bovine brucellosis. Several conventional serological tests have been used as a single or in combination for the diagnosis of this disease (FAO/WHO, 1986). A rapid screening test of high sensitivity is usually applied initially for the testing of sera in control programs. A positive reaction in the screening test would result in the serum being tested in a confirmatory test of higher specificity.

In most countries, agglutination tests such as the Rose Bengal plate test (RBPT) and the buffered plate antigen test (BPA) are currently used as screening tests, while other agglutination tests such as the 2mercaptoethanol test (2ME), the Rivanol test (RIV), the EDTA modified SAT and the slow tube agglutination test (SAT) are regularly used as confirmatory tests. However, the agglutination techniques may have limitations in sensitivity resulting from prozoning (Alton et al., 1975) or non-specificity because of cross-reactivity. In the first case, the result can be missed detection of positive animals and, in the second, false-positive reactors are detected due to the presence of common antigenic determinants between B. abortus and several other bacteria (Hurvell and Lindberg, 1973; Corbel, 1975 and Marino et al., 1991).

The diagnosis of brucellosis in cattle is frequently complicated particularly when live vaccines such as strain 19 of $B$. abortus are used on a large scale. The occurrence and persistence of serum antibodies following Brucella strain 19 vaccination is the major disadvantage since antibodies may interfere with detection of brucellosis infected cattle. The antibody response induced by these vaccines is difficult to distinguish from that of natural infection by conventional serological tests (FAO/WHO, 1971). Several supplementary serological tests such the 
agglutination with 2 mercaptoethanol, complement fixation and agar-gel immunodiffusion containing a soluble polysaccharide antigen have been shown to differentiate to some extent the antibody response of vaccinated from infected animals (Diaz et al., 1979; Alton et al., 1988).

In the recent past, indirect enzyme linked immunosorbent assay (iELISA) and competitive enzyme linked immunosorbent assay (cELISA) have been much popular and extensively used. Despite excellent performance and superiority of iELISA over conventional serological tests for diagnosing brucellosis in various species of animals the assay is not able to distinguish animals infected with virulent Brucella or vaccinated with $B$. abortus strain 19 vaccine. The cELISA is a multi-species assay which has the ability to determine the antibody to B. abortus in various species of animals and generally do not react with sera containing residual antibody from vaccination with B. abortus strain 19. However, in Egypt B.melitensis organism is the main isolate from different species of animals infected with Brucella (Refai, 2002). Therefore, we must distinguish between antibodies due to infection with $B$. melitensis and that of B. abortus strain 19 vaccine.

The objective of the present study was to validate the diagnostic performance characteristics of the competitive ELISA to the indirect ELISA and to the standard serological techniques on sera from negative, infected not vaccinated and B. abortus strain 19 vaccinated-infected cattle.

\section{MATERIALS and METHODS}

\section{Test Sera:}

Negative sera: A total of 270 sera were obtained from herds in an area without recent history of infection or vaccination against brucellosis.

Positive sera: A total of 540 serum samples were obtained from herds from which $B$. melitensis was isolated. These sera were segregated into two groups:

1- 360 sera from herds infected not vaccinated with Brucella strain 19 vaccine.

2- 180 serum samples from vaccinated cows with Brucella strain 19 vaccine which showing some abortions and reproductive disorders.

\section{Serological Tests:}

\section{1- Conventional tests:}

The tests used were the RBPT and the SAT as described by (Alton et al., 1988) and the EDTA modified SAT according to (MacMillan, 1990). In the RBPT any degree of agglutination was considered to be 
positive. For the SAT, visible agglutination at the dilution of $1 / 40++$ or more was considered to be positive and for the EDTA modified SAT, visible agglutination at the dilution of $1 / 10$ was considered to be positive.

\section{2- Indirect ELISA:}

An ELISA kit (Brucelisa) provided by the VLA, (an executive agency of the Department for Environment, Food and Rural Affairs), which contained all the necessary reagents was used. The test was performed according to the manual which is accompanied with the kit. Briefly, a primary dilution of $1 / 40$ of all test and control sera were made by adding $25 \mu \mathrm{l}$ of serum to $1 \mathrm{ml}$. of diluting buffer (5 Tablets of Phosphate Buffer Saline (PBS), $0.5 \mathrm{ml}$ of phenol red indicator and $250 \mu \mathrm{l}$ of Tween 20 to $500 \mathrm{ml}$ of distilled water). The plate was prepared by adding $80 \mu \mathrm{l}$ of diluting buffer to all wells. Then, $20 \mu \mathrm{l}$ of each of primary diluted samples were added to all prepared wells except columns 11 and 12 (this gives a final dilution of 1/200). Twenty microns of the primary diluted positive control were added to each of the wells in column 11 and $20 \mu 1$ of the primary diluted negative control were added to each of the wells in column 12 except $\mathrm{H} 12$ which should have no sample added as it is to be used to blank the plate. Then, the plate was covered with the lid and incubated at room temperature for 30 minutes on a rotary shaker. The contents of the plate were shaked out and rinsed 5 times with washing solution (one ampoule of $\mathrm{Na}_{2} \mathrm{HPO}_{4}$ and $1 \mathrm{ml}$ of Tween 20 to 10 liters of distilled water) and then thoroughly dried by tapping the plate on absorbent paper towel. The diluted conjugate solution, $100 \mu \mathrm{l}$ were added to all wells. The plate was covered with a lid and incubated for 30 minutes on a rotary shaker at $160 \mathrm{revs} / \mathrm{min}$. Then the plate was shaked out, rinsed 5 times with washing solution and then thoroughly dried (as previously described). Microplate reader was switched on and allowed to be stabilized for 10 minutes. A hundred microns of substrate solution $(300 \mu \mathrm{l}$ of ABTS chromogen to $12 \mathrm{ml}$. of substrate buffer plus $60 \mu \mathrm{l}$ of substrate (hydrogen peroxide) were added to each well and the plate was left at room temperature for a minimum of 10 minutes and a maximum of 15 minutes. To each well, $100 \mu \mathrm{l}$ of stopping solution (one ampoule of sodium azide with $500 \mathrm{ml}$ of distilled water) were added, condensation of the bottom of the plates was removed with an absorbent paper towel. Finally the pate was read at $450 \mathrm{~nm}$ blanked on well $\mathrm{H} 12$.

Analysis of the results: Colour development within a well indicated that the sample tested had antibodies to Brucella. A positive/negative cut-off was calculated as $10 \%$ of the mean of the optical density (OD) of the 8 
positive control wells. Any test sample giving an OD equal to or above this value should be considered as being positive.

\section{3-Competitive ELISA:}

An ELISA kit (Compelisa) provided by the VLA, (an executive agency of the Department for Environment, Food and Rural Affairs), which contained all the necessary reagents was used. The test was performed according to the manual which is accompanied with the kit. Briefly, the conjugate solution was prepared and diluted to working strength with diluting buffer (5 tablets of PBS), $0.5 \mathrm{ml}$ of phenol red indicator and $250 \mu \mathrm{l}$ of Tween 20 to $500 \mathrm{ml}$ of distilled water). For each tested serum, $20 \mu \mathrm{l}$ were added per well. The columns 11 and 12 were left as controls of which $20 \mu \mathrm{l}$ of the negative one were added to wells $\mathrm{A} 11, \mathrm{~A} 12, \mathrm{~B} 11, \mathrm{~B} 12, \mathrm{C} 11$ and $\mathrm{C} 12$, while $20 \mu \mathrm{l}$ of the positive one were added to wells F11,F12,G11,G12,H11 and H12. The remaining wells of the columns 11 and 12 that have no serum added, act as the conjugate controls. To all wells, $100 \mu \mathrm{l}$ of the prepared conjugate solution were dispensed which give a final serum dilution of $1 / 6$. Then the plate was vigorously shaken on the microtitre plate shaker for 2 minutes. The plate was covered with the lid and incubated at room temperature $\left(21^{\circ} \mathrm{C} \pm 6{ }^{\circ} \mathrm{C}\right)$ for 30 minutes on a rotary shaker at $160 \mathrm{revs} / \mathrm{min}$. The contents of the plate were shaked out and rinsed 5 times with washing solution (one ampoule of $\mathrm{Na}_{2} \mathrm{HPO}_{4}$ and $1 \mathrm{ml}$ of Tween 20 to 10 liters of distilled water) and then thoroughly dried by tapping on absorbent paper towel. The microplate reader was switched on and allowed the unite to be stabilized for 10 minutes. The substrate and chromogen solutions were prepared immediately before use (One tablet of urea $\mathrm{H}_{2} \mathrm{O}_{2}$ in $12 \mathrm{ml}$ of distilled water, when dissolved the OPD tablet was added and mixed thoroughly) of which $100 \mu 1$ were added to each well. Then, the plate was left at room temperature for a minimum of 10 minutes and a maximum of 15 minutes. Slowing the reaction by adding $100 \mu \mathrm{l}$ of stopping solution to all wells (One ampoule of citric acid with $38 \mathrm{ml}$ of distilled water). Condensation of the bottom of the plate was removed with absorbent towel and then the plate was read at $450 \mathrm{~nm}$.

Analysis of the results: The lack of colour development indicated that the sample tested was positive. A positive/negative cut-off was calculated as $60 \%$ of the mean of the optical density (OD) of the 4 conjugate control wells. Any test sample giving an OD equal to or below this value should be regarded as being positive.

Sensitivity and Specificity: The sensitivity of tests used on sera from infected cattle and the specificity in brucellosis free animals was 
calculated according to Thrusfield (1986) and followed the formulae for each test:

$$
\begin{gathered}
\text { Sensitivity }=\begin{array}{c}
\text { Total number of positive results } \\
\text { Total number of positive animals sampled }
\end{array} \\
\text { Specificity }=\quad \begin{array}{c}
\text { Total number of negative results } \\
\text { Total number of negative animals sampled }
\end{array}
\end{gathered}
$$

\section{Bacteriological Evaluation:}

Milk samples $(n=130)$ were collected under sterile conditions (30 samples from Brucella free animals, 40 samples from infected not vaccinated ones and 80 samples from vaccinated cows showing some abortions and reproductive disorders. All samples were cultured in selective solid media and the suspected isolates were identified as B. melitensis according to MacMillan (1990).

\section{RESULTS}

\section{Negative sera:}

The specificities of the five serological tests performed on sera from 270 brucellosis-free cows are presented in Table (1).

\section{Positive sera:}

1- The sensitivities of the five serologic tests performed on 360 Brucellainfected not vaccinated animals are presented in Table (2).

2- The specificity of the five serological tests performed on 180 vaccinated Brucella-infected animals are presented in Table (3).

Table 1: The Specificity of Different Tests on 270 Sera From BrucellosisFree Cows.

\begin{tabular}{lccc}
\hline Test & Negative & Positive & Specificity \\
\hline RBPT & 270 & 0 & $100 \%$ \\
SAT & 268 & 2 & $99.2 \%$ \\
EDTA-m-SAT & 270 & 0 & $100 \%$ \\
iELISA & 265 & 5 & 98.1 \\
cELISA & 270 & 0 & $100 \%$ \\
\hline
\end{tabular}

RBPT=Rose Bengal Plate Test 
SAT $=$ slow tube agglutination test

EDTA-m-SAT $=$ EDTA modified SAT

iELISA=-Indirect ELISA

cELISA=Competitive ELISA

Table 2: The Sensitivity of Different Serologic Tests on 360 Sera from Brucella-Infected Not Vaccinated Cows.

\begin{tabular}{lccc}
\hline Test & Positive & Negative & Sensitivity \\
\hline RBPT & 360 & 0 & $100 \%$ \\
SAT & 304 & 56 & $84.4 \%$ \\
EDTA-m-SAT & 339 & 21 & $94.1 \%$ \\
iELISA & 354 & 6 & $98.3 \%$ \\
cELISA & 350 & 10 & $97.2 \%$
\end{tabular}

(See Table 1 for key)

Table 3: The Specificity of Different Serological Tests on 180 Sera from Strain 19 Vaccinated- Infected Cows.

Test Positive Negative Specificity

$\begin{array}{lccc}\text { RBPT } & 20 & 160 & 88.8 \% \\ \text { SAT } & 31 & 149 & 82.7 \% \\ \text { EDTA-m-SAT } & 11 & 169 & 93.8 \% \\ \text { iELISA } & 50 & 130 & 72.2 \% \\ \text { cELISA } & 4 & 176 & 97.7 \%\end{array}$

(See Table 1 for key)

\section{Bacteriological Results:}

Sixteen Br.melitensis biovar 3 were isolated from 130 milk samples where no isolates from Brucella free animals, 12 isolates from infected not vaccinated ones and 4 isolates from vaccinated cows showing some abortions and reproductive disorders. Sera from animals from which by Alton et al. (1988) were isolated showing positive results in all the above mentioned serological tests. 


\section{DISCUSSION}

A simple, rapid and inexpensive serological test that will detect infected animals early in the incubation period and at all stages of the disease and that does not detect antibody in vaccinated animals is still to be found. Nevertheless, a great deal of improvement was achieved recently either by the introduction of enzyme immunoassays (Wright et al., 1990) or by vaccination of animals with reduced dose of Strain 19 B. abortus vaccine (Plommet and Fensterbank, 1984).

In the present study two different enzyme immunoassays were compared with conventional tests on sera of negative, infected not vaccinated and vaccinated-infected animals. The specificity of the enzyme immunoassays was high, $98.1 \%$ for the iELISA and $100 \%$ for the cELISA on sera from brucellosis-free animals (Table 1). In this situation, the iELISA was the test which gave the highest rate of false positive reactions (Nielsen, 1995; Saravi et al., 1995; OIE, 1996).

The discrepancy between the higher number of reactors detected by RBPT than the other tests (Table 2) is due to the fact that RBPT is a highly sensitive test which can detect low titre as in cases of chronic brucellosis, that not be considered as positive by the quantitative tests. (Nicoletti and Milward, 1983).

From the above mentioned results, the difference between SAT and EDTA modified SAT in such sera are though to contain IgM molecules which cause agglutination of B.abortus test antigen in a nonimmune manner, namely by binding by $\mathrm{Fc}$ region. This binding is inhibited by EDTA, while specific binding by the IgM, Fab region (induced by infection with Brucella organisms) is unaffected (MacMillan, 1990). The sensitivities of the ELISAs were $98.3 \%$ for the indirect and 97.2\% for the competitive in infected not vaccinated animals (Table 2). Except for the RBPT, the two ELISAs were more sensitive than the other tests. These findings are in accordance with others (Sutherland, 1984; Dohoo et al., 1986; Uzal et al., 1996; Al-Farwachi et al., 2009) in that RBPT was found to be a good screening test, although some authors (Saravi et al., 1990) have found an unacceptable false negative rate with the RBPT.

In the vaccinated -infected group (Table 3), the iELISA was the most sensitive test, giving a high percentage of positive results. This may be explained by the use of a polyclonal antibovine $\operatorname{IgG}(\mathrm{H}+\mathrm{L})$ conjugate which measures all isotypes present in the sera. On the other hand, the competitive ELISA with $B$. melitensis coated plate differentiates 4 animals, from which B.melitensis organisms were isolated, from Brucella 
strain 19 vaccinated group. Some authors speculate that it can be a result of antigen presentation on the test and the antibody affinity (Wright $e t$ al., 1990; Nielsen et al., 1992; Uzal et al., 1996; Aguirre et al., 2002; Chand and Puran, 2006).

The results presented above raise the conclusion, it is advisable to use the RBPT as a screening test and cELISA as a confirmative test in those areas where strain 19 vaccination is routinely applied. On the other hand in areas free of vaccination perhaps the most advisable test as a confirmative one could be the iELISA because of its ability to detect small amount of IgG1.

\section{ACKNOWLEDGEMENTS}

The authors wish to thank to the Veterinary Laboratories Agency (VLA), for its helpful assistance by providing the two ELISAs kits used in this research. The VLA is an Executive Agency of the Department for Environment, Food and Rural Affairs (New Haw, Addlestone, Surrey KT15 3NB Untied Kingdom). Also, The authors kindly would like to thank Egyptian -Spanish Project for control of Ruminant Brucellosis in the upper Egypt for their helpful in collecting samples from vaccinated animals and their confirmation for the isolated strains in this research in their Brucella Lab. in Cita, Espain.

\section{REFERENCES}

Aguirre, N.P.; Vanzini, V.R.; Torioni de Echaide, S.; Valentini, B.S.; De Lucca, G.; Aufranc, C.; Canal, A.; Vigliocco, A. and Nielsen, K. (2002): Antibody dynamics in Holestein Friesian Heifers vaccinated with Brucella abortus strain 19, using seven serological tests. J.Immunoassay and Immunochemistry. 23 (4): 471-478.

Al-Farwachi, M.I.; Al-Iraqi, O.M.; Al-Hankawe, O.Kh. and AbdulMajeed, M.O. (2009): Using of competitive ELISA in detection of brucella antibodies in cattle sera in Mosul city. Iraqi J. of Vet. Sci. 23(2): 97-103.

Alton, G.G.; Jones, L.M. and Pietz, D.E. (1975): Laboratory techniques in brucellosis. 2nd. ed. «FAO-WHO, Geneva Series de monograffa.

Alton, G.G.; Jones, L.M.; Angus, L.D. and Verger, J.M. (1988): Techniques for the brucellosis laboratory, Paris, Institut National de la Recherche Agronomique. 1988 pp. 190 pp. 
Chand, P. and Puran, C. (2006): Competitive ELISA for the diagnosis of brucellosis in cattle. J. of Immunology and Immunopathology. 8 (2): 0972-0561. Haryana Agricultural University, Hisar, India.

Corbel, M.J. (1975): The serological relationship between Brucella sp., Yersinia enterocofitica serotype IX and Salmonella serotypes of Kaufman-White group, N. J. Hyg, 75: 151-171.

Diaz, R.; Garatea, P.; Jones, L.M. and Moriyon, I. (1979): Radial immunodiffusion with a Brucella polysaccharide antigen for differentiating infected from vaccinated cattle. J. Clin. Microbiol., 10: 37-41.

Dohoo, I.R.; Wright, P.F.; Ruckerbauer, G.M.; Samagh, B.S.; Robertson, F.J. and Forbes, L.B. (1986): A comparison of five serological tests for bovine brucellosis, Can. J. Vet. Res., 50: 485-493.

FAO/WHO, (1971): Expert Committee on Brucellosis, Fifth Report, WHO Technical Report, Series464, Agricultural Studies, Geneva, N.85. FAO/WHO, (1986): Expert Committee on Brucellosis, 6th Report, World Health Organization, Technical Report, Series 740.

Hurvell, B. and Lindberg, A.A. (1973): Serological cross-reactions between different Brucella species and Yersinia enterocolitica. Immunochemical studies on phenol-water extracted lipopolysaccharides from Brucella abortus and Yersinia enterocolitica type IX.Acta Pathol Microbiol Scand B Microbiol Immunol. 81(1): 113-119.

Macmillan, M. (1990): New answers to old questions: what the complete Freud-Fliess correspondence tells us. Psychoanal Rev., 77(4): $555-572$.

Marino, O.; Galleco, M.M.I.; De Leon, L.S. and Almansa, M.J. (1991): Comparaci6n $d$ and $t \sim$ cnicas serologicas en la evaluadon de bovinos infectados naturalmente por Bruceila abo fins. En: J. FRANK (eds.). Networking in Brucellosis Research. The United Nations University, pp 120-130.

Nicoletti, P. and Milward, F.W. (1983): Protection by oral administration of Brucella abortus strain 19 on oral challenge exposure with a pathogenic strain of Brucella. Am. J. Vet. Res., 44(9): 1641-3.

Nielsen, K.H.; Gall, D.; Kelly, W.; Henning, D. and Garcia, M. (1992): Enzyme immunoassay, Application to diagnosis of bovine brucellosis, ADRI, Agriculture Canada.

Nielsen, K.H. (1995): A brief review of diagnosis of bovine brucellosis by detection of antibody. Arch. Med. Vet., 27: 39-43. 
OIE, (1996): Office International des Epizooties, World organization for animal health, Manual of Standards for Diagnostic Test and Vaccines, Chapter 3.2.1 Bovine Brucellosis, Third Edition.

Plommet, M. and Fensterbank, R. (1984): La vaccination antibrucellique administrée par voie conjonctivale, Dev. Biol. Stand., Paris 56: 681-687.

Refai, M. (2002): Incidence and control of brucellosis in the Near East region. Vet.Microbiol., 90: 81-110.

Saravi, M.A.; Gregoret, R.J.; Wright, P.F. and Gall, D. (1990): Comparison of an indirect enzyme immunoassay and conventional serological techniques for the detection of bovine antibody for Brucella abortus, Proc. Final Res. Coord. Meet. FAO/IAEA/SIDA, Austria, 151-162.

Saravi, M.A.; Wright, P.F.; Gregoret, R.J. and Gall, D.E. (1995): Comparative performance of the enzyme-linked immunosorbent assay (ELISA) and conventional assays in the diagnosis of bovine brucellosis in Argentina.Vet. Immunol. Immunopathol., 47(1-2): 93-99.

Sutherland, S.S. (1984): Evaluation of the Enzyme-Linked Immunosorbent Assay in the detection of cattle infected with Brucella abortus, Vet. Microbiol., 10: 23-32.

Thrusfield, M. (1986): Veterinary Epidemiology, Butterworth, London, First edition.

Uzal, F.A.; Carrasco, A.E.; Nielsen, K.; Echaide, S. and Cabrera, R.F. (1996): An indirect ELISA using a monoclonal anti IgG1 enzyme conjugate for the diagnosis of bovine brucellosis. Vet. Microbiol., 52 (1-2): 175-180.

Wright, P.; Nielsen, K.H. and Kelly, W. (1990): Primary binding techniques for the serodiagnosis of bovine brucellosis, In: Animal Brucellosis, Nielsen, K.H. and Duncan, J.R. (Ed), CRC Press, Boca Raton, pp 199-235. 